\title{
Continuous Flow Manufacturing for API: From Potential Evaluation to Production Scale Realization
}

\author{
D. KIRSCHNECK
}

Microinnova Engineering GmbH, Reininghausstrasse 13a, 8020 Graz, Austria

E-mail: Dirk.Kirschneck@microinnova.com

Sci Pharm. 2010; 78: 668

doi:10.3797/scipharm.cespt.8.PMS41

\begin{abstract}
Process performance in the synthesis of API and its intermediates can be reached through new technologies and methods. Significant development time reduction, operating cost reduction by yield improvement, energy savings and a fundamental improvement of plant safety are some of the main results of process intensification concepts. The activities can be divided into 4 phases: 1. evaluation, 2. verification, 3. development and 4. realization. A riskbased approach according to ICH Q9 is used during the whole project.

First of all the identification of reaction performance potential of existing chemical plants needs to be analyzed ending with the formulation of a target for the verification (basic feasibility). The evaluation starts with a process capability check, followed by the generation of a cost flow analysis and ends with a technology evaluation and comparison. Microinnova compares the needs for a chemical reaction with technology profiles to find out the most suitable solution for a process.

In the second phase the intensification potential of the reaction needs to be verified. Typically a continuous plant setup is developed and modified according to the needs of the chemical process. An operating procedure will be generated and optimized. Finally, a rough parameter scan of a few key parameters will deliver a relevant overview for the verification.

Once the potential is proven the process development needs to be done, where the basic feasibility delivers a good basis for the work (phase 3 ). The reaction process will be optimized. New process options are used as well as new technology concepts like microreactors and flow chemistry to find the best possible process. A knowledge based approach according to $\mathrm{ICH}$ Q8 will be used for the development by applying methods like the critical parameter concept and cause effect chains. Engineering data necessary for the scale up will be generated in this part of the work.

Finally in the fourth phase the observed performance needs to be realized. The deep specific knowledge in process intensification equipment needs to be combined with process experience and competence. The best possible equipment solution needs to be selected and calculated under considerations regarding the common standards like ATEX or cGMP. The gap between continuous processing and multi-purpose flexibility can be bridged by modularization of continuous plants.
\end{abstract}

